\title{
Rapid micro-immunohistochemistry
}

\author{
Robert D. Lovchik', David Taylor (10 ${ }^{1,2}$ and Govind Kaigala (1)
}

\begin{abstract}
We present a new and versatile implementation of rapid and localized immunohistochemical staining of tissue sections. Immunohistochemistry $(\mathrm{IHC})$ comprises a sequence of specific biochemical reactions and allows the detection of specific proteins in tissue sections. For the rapid implementation of $\mathrm{HC}$, we fabricated horizontally oriented microfluidic probes (MFPs) with functionally designed apertures to enable square and circular footprints, which we employ to locally expose a tissue to time-optimized sequences of different biochemicals. We show that the two main incubation steps of IHC protocols can be performed on MDAMB468-1510A cell block sections in less than $30 \mathrm{~min}$, compared to incubation times of an hour or more in standard protocols. IHC analysis on the timescale of tens of minutes could potentially be applied during surgery, enabling clinicians to react in more dynamically and efficiently. Furthermore, this rapid $\mathrm{IHC}$ implementation along with conservative tissue usage has strong potential for the implementation of multiplexed assays, allowing the exploration of optimal assay conditions with a small amount of tissue to ensure high-quality staining results for the remainder of the sample.
\end{abstract}

\section{Introduction}

Immunohistochemistry (IHC) is a well-established technique to detect the spatial distribution of antigens in tissue sections and is now routinely used for tumor analysis in research laboratories and diagnostic centers. Interestingly, this method has been used for well over 50 years with limited modifications to the underlying principle, owing to its robustness. In contrast, it has undergone several innovations in implementation, automation and throughput. With each innovation, user input has been reduced, but often at the expense of flexibility and versatility.

After sample-dependent pretreatment, in the first step of an IHC assay, a primary antibody, which specifically binds to an antigen of interest, is applied to the tissue. In the second step, a secondary antibody is applied. The secondary antibody, which helps to amplify the detection signal, is usually polyclonal and conjugated with enzymes that enable subsequent detection of the signal through

\footnotetext{
Correspondence: Govind Kaigala (gov@zurich.ibm.com)

${ }^{1}$ IBM Research Europe, Saeumerstrasse 4, 8803 Rueschlikon, Switzerland.

${ }^{2}$ Present address: Eidgenössische Technische Hochschule Zürich, Department of Mechanical and Process Engineering, Sonneggstrasse 3, 8092 Zurich, Switzerland
}

colorimetric reactions. This series of incubation steps allows antigen localization through the choice of antibody and control over the reaction kinetics, thus aiding semiquantitative or quantitative estimation of the amounts of antigen present in the sample. The commercially sourced antibodies in these protocols often vary in their specificity and sensitivity, thus requiring meticulous optimization and testing ${ }^{1}$. Hence, there is a need to enable users to rapidly screen parameter spaces to determine practical assay conditions for the specific biochemicals used. The ability to quantitatively characterize detected signals, perform multiplexed detection of various antigens simultaneously, and rapidly implement IHC protocols that are standardized or modified according to user need are facets of research that would be essential to fostering the next generation of methods in cancer research and diagnostics.

Several notable efforts have been made to enable a quantitative proteomic assessment of tissue sections via IHC, for example, Dupouy et al. ${ }^{2}$, Carvajal-Hausdorf et $\mathrm{al}^{3}$, and Xing et al. ${ }^{4}$. In our previous work, Kashyap et al. $^{5}$, using a microfluidic probe, we introduced a method for the implementation of IHC as a quantitative assay ( $\mathrm{q} \mu \mathrm{IC}$ ). Our $\mathrm{q} \mu \mathrm{IC}$ method looks at the evolution of the signal strength to extract quantitative information. 
The current design of the MFP devices that we present here could also be used for $q \mu \mathrm{IC}$. To broaden the applicability of the MFP, this work shows how we increased the rapidity of IHC and implemented sequential chemistry in small and defined regions of tissues. Significant improvements in the speed and reproducibility of these assays remain essential for rendering quantitative IHC useful in a routine clinical context.

Numerous methods have also been proposed for implementing multiplexed IHC analysis. These are broadly classified as follows: 1. Application of different primary antibodies to the same region of a tissue. To avoid cross-reactivity in the detection step, primary antibodies must be produced in different species, which greatly limits the number of antigens that can be detected simultaneously ${ }^{4}$. This constraint can be relaxed by stripping antibodies deposited in a previous analysis and by bleaching the resulting detection signal prior to performing a subsequent test. This sequential stripping and bleaching is laborious, time-consuming and, importantly, tends to deteriorate the tissue. 2. The layered peptide arrays technique, where the antigens are blotted onto several layers of stacked membranes, which allows independent analysis of each membrane subsequently while preserving the spatial information of the obtained IHC data $^{6}$. Although suitable for multiplexing, this approach is complex and consumes the tissue section entirely. 3 . Spatial segregation to present different primary antibodies on a tissue section, which allows the use of any type of antibody on neighboring reaction sites ${ }^{7}$. Depending on the resolution of the spatial confinement of the corresponding antibody solutions, this allows the mapping of specific regions of interest (ROIs) in a multiplexed manner. This implementation overcomes several of the limitations of other implementations described above by obviating harsh chemical stripping or adding complexity to the IHC protocol.

One potential strategy for spatially segregated multiplexing is to implement co-flow laminar streams of antibody solutions in open-top microchannels pressed onto a tissue section, as reported by Kim et al. ${ }^{8}$ and Ciftlik et al. ${ }^{9}$. The required mechanical contact with the tissue sections nevertheless consumes parts of the sample and offers limited flexibility in terms of addressing specific ROIs on the tissue section, which becomes critical when the ROIs are very small in comparison with the total tissue area. A versatile and IHC-compatible strategy is to localize antibody solutions in a so-called hydrodynamic flow confinement (HFC) formed at the apex of a noncontact liquid scanning probe, such as a microfluidic probe (MFP) ${ }^{10}$. Multiplexed IHC routines using an MFP were demonstrated by Lovchik et al. ${ }^{7}$ and Queval et al. ${ }^{11}$ at the micrometer scale and expanded to the centimeter scale by Taylor et al. ${ }^{12}$. Such flow-based approaches were also used for brain slice microenvironment modification ${ }^{11}$ and breast cancer sections $^{12}$, as well as in combination with lanthanide-based immunocomplexes ${ }^{13}$ and immunofluorescence ${ }^{14}$.

Another important aspect pertaining to IHC analysis is the total time required to implement the complete assay. In traditional $\mathrm{IHC}$, the incubation time of each antibody is 30-60 min, sometimes longer, even overnight. This step significantly contributes to the total time required for an IHC test. In earlier work, we presented micrometer-scale $\mathrm{IHC}^{7}$, in which we implemented the highly localized delivery of primary antibodies on tissue sections with an MFP. The time required for the presentation of the primary antibody to each area of the section was in the range of $\sim 30-60 \mathrm{~s}$. This microfluidic approach, allowing a reduction in the incubation times, was enabled by two advantages: (a) only small volumes of liquids are consumed, allowing the concentration of antibodies to be increased without increasing the cost of the assay, and (b) the flow of the antibody solution allows the continuous replenishment of the antibodies consumed from the solution, thereby making the reaction time largely independent of the relatively low diffusion coefficients of the antibodies. Apart from our demonstration of expanding MFP-assisted IHC from the micrometer scale to the centimeter scale, Taylor et al. ${ }^{12}$, a related publication by Cors et al. ${ }^{15}$ additionally showed the integration of dewaxing and rehydration with an MFP to further increase the rapidity of multiplexed IHC staining.

The challenge faced in our and others' prior work was that processing the whole slide for the standard IHC workflow, with the MFP performing only one of the steps, made later use of the slides for other assays challenging. In this paper, we focus on taking advantage of an MFP to boost the rapidity of the assay by enhancing the reaction rates and automation by rapid switching between different types of processing liquids, allowing the fast implementation of sequential assay steps (see Fig. 1). We further demonstrate different ways of performing sequential chemistry with an MFP to integrate the whole workflow, such that other assays might be run concomitantly, prior to any whole slide processing. The rapidity of the assay and localization of the applied liquids allow efficient optimization of the processing parameters, using only a small fraction of the tissue. We designed a new horizontally oriented MFP head with apertures that enable the implementation of arbitrarily shaped footprints (see Fig. 2). Radial and square-shaped flow confinements were chosen, which are well suited for sequential presentation of different liquids on surfaces from the micrometer to millimeter scale. Such radial or square-shaped HFCs are formed by the central injection of a processing liquid, which is reaspirated by surrounding ring- or squareshaped apertures. We chose to locally implement the 


\section{Workflow of conventional IHC}

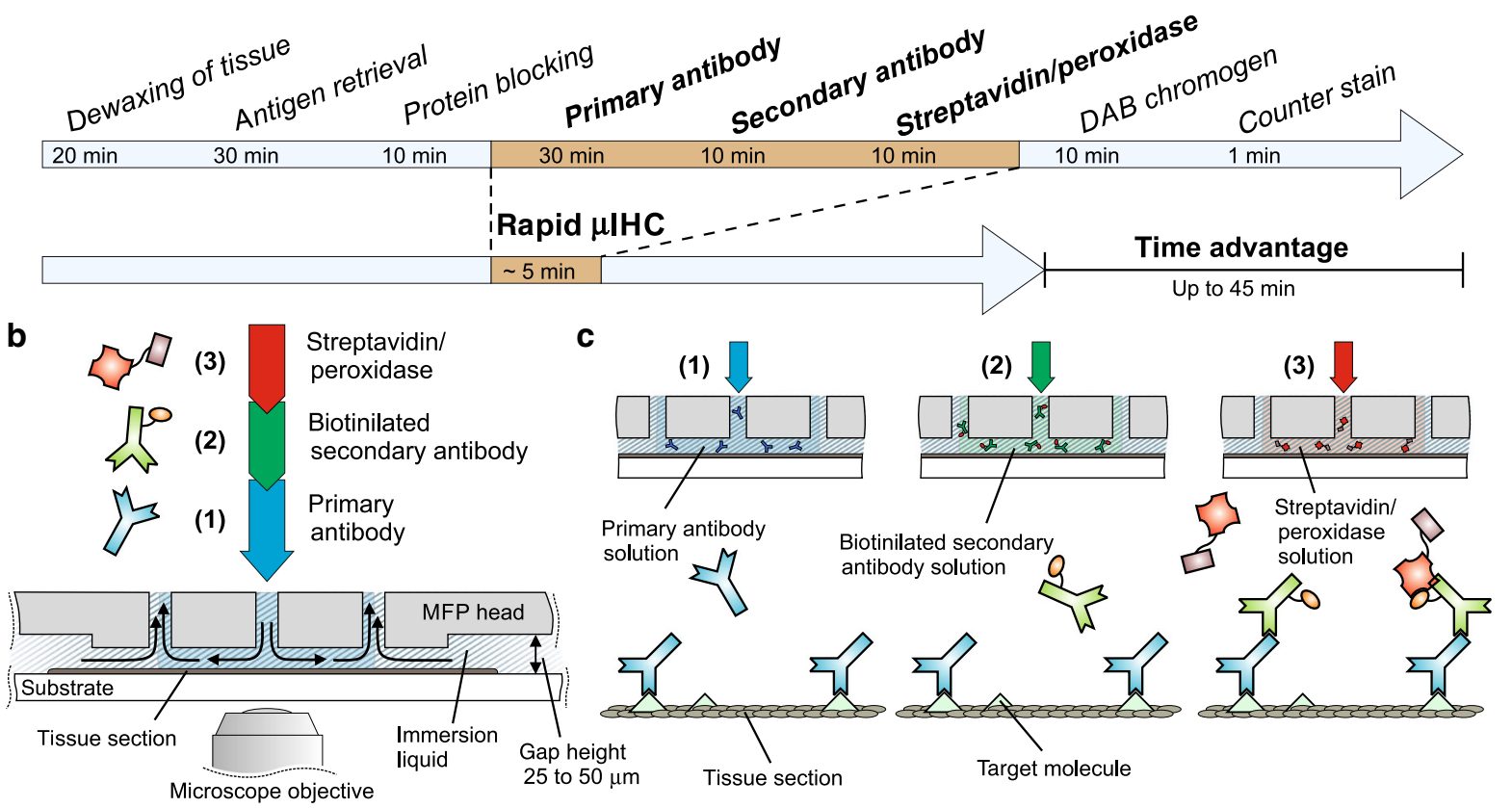

Fig. 1 Illustration of the steps involved inImmunohistochemistry and its implementation using an MFP. a Protocol for immunohistochemistry implemented with chromogen-based revelation. Rapid $\mu \mathrm{HC}$ is enabled by implementing the three core steps of the IHC protocol using an MFP. The times shown for the individual steps of the conventional workflow are taken from the suggested protocol of the Mouse-specific HRP/DAB Detection Kit from Abcam (ab64259). b Scheme of three different reagents passing through the central aperture of an MFP head placed over a substrate. The central injection aperture is linked to three channels through which the reagents are injected sequentially. $\mathbf{c}$ The three core steps of rapid $\mu \mathrm{lHC}$ are (1) the binding of a primary antibody to an antigen on the sample, (2) the binding of a biotinylated secondary antibody to the primary antibody, and (3) the application of a streptavidin/peroxidase solution for visualization

three core steps of an IHC routine using horizontal probe heads: the application of the primary antibody, the secondary antibody and the enzyme (peroxidase). These three steps largely determine the quality of the obtained stain and usually require from $30 \mathrm{~min}$ up to several hours in on-bench protocols. Owing to convection-enhanced deposition and the possibility of using higher concentrations of antibodies without increasing the overall consumption of reagents, the MFP is well suited to locally implement these core steps of IHC analysis within minutes; we refer to this method as "rapid $\mu \mathrm{IHC}$ ".

\section{Theory}

The most important steps in IHC staining protocols are the incubation of the tissue with primary and secondary antibodies. Here, we show the dependency of the IHC signal on multiple parameters. The amount of antibodies binding to receptors on the surface of the sample $b(t)$ depends on several parameters. The value of interest, to be semiquantitatively assessed by an IHC staining routine, is the surface density of binding sites $b_{m}$. Further factors that can be adjusted to optimize the assay conditions of an MFP-based staining protocol are the composition of the buffer, the temperature $T$, the bulk concentration of the antibodies in the processing liquid $c_{0}$, the flow velocity of the processing liquid (a function of the injection and aspiration flow rates, $Q_{i}$ and $Q_{a}$, and the gap distance $d$ ),

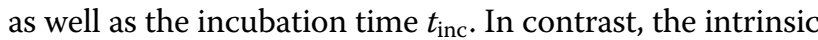
properties of the applied antibodies, namely, the association and dissociation constants with their respective target $k_{\mathrm{on}}$ and $k_{\mathrm{off}}$ and their diffusivity $D_{\mathrm{ab}}$, are not directly optimizable and are subject to significant batch-to-batch variations and variability caused by varying storage conditions and other factors ${ }^{16,17}$.

To estimate the impact of a change in $k_{\text {on }}$, e.g., of the primary antibody, we assume that, due to convection inherent to the MFP, the binding of antibodies to the sample is reaction limited and $D_{\text {ab }}$ can, therefore, be neglected. We also assume that all the involved species are first-order reactants, and the receptor ligand binding reaction at the sample, therefore, follows a Hill-Langmuir characteristic. In such a scenario, the rate of the surface reaction is

$$
\frac{\mathrm{d} b}{\mathrm{~d} t}=k_{\mathrm{on}} \cdot c \cdot\left(b_{\mathrm{tot}}-b\right)-k_{\mathrm{off}} \cdot b
$$


a

MFP head and holder assembly

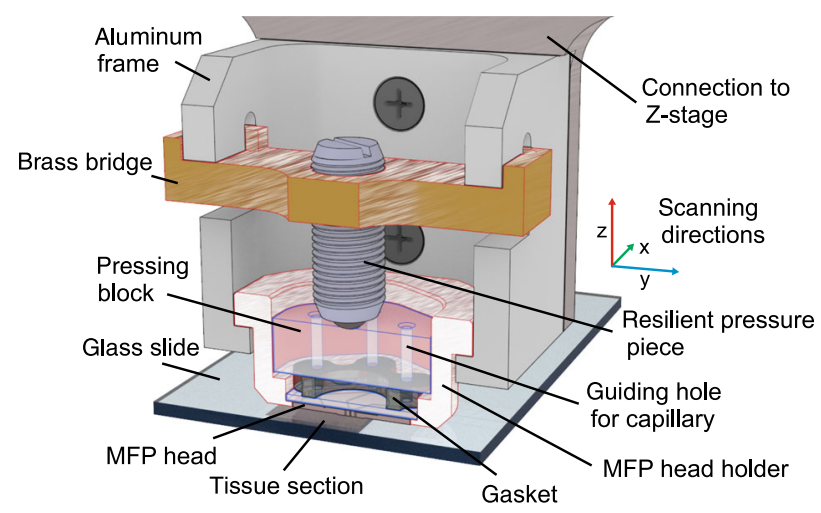

b Horizontal silicon/glass MFP heads

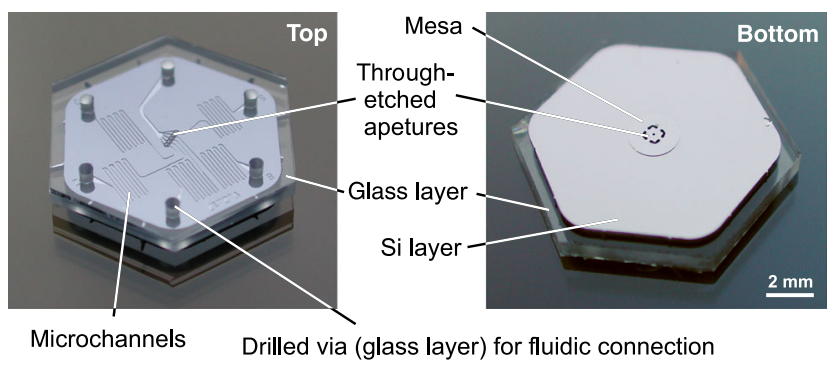

C

FE simulations of flow confinements

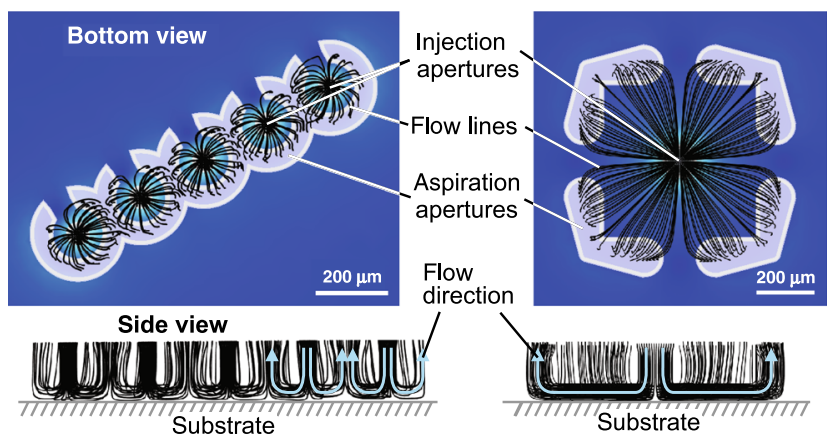

Fig. 2 The holder assembly, single units of horizontal microfluidic probe heads, and (corresponding) FE simulations of flow confinements. a Assembly of the MFP head holder: the MFP head is seated on its glass overhang within an aluminum holder. A molded PDMS gasket for holding and sealing up to six capillaries is placed on top of the head, and a mechanical compression force is applied to the gasket using a resilient pressure piece and a pressure block. All components of the assembly are held by an aluminum frame that is mounted onto the Z-stage of the scanning unit. $\mathbf{b}$ Photographs of horizontal MFP heads made of Si and glass. The top-view shows the vias drilled through the glass layer for fluidic connection and the routing of the microchannels for addressing the apertures of the head. The mesa and the injection/aspiration apertures can be seen on the bottom view. The height of the mesa is typically $50 \mu \mathrm{m}$, and the diameter varies between 2 and $4 \mathrm{~mm}$, depending on the aperture designs. c FE simulations were used to study injection/aspiration ratios for different HFC geometries at varying heights

Integration of Eq. (1) yields the amount of primary antibodies bound to the sample after a given time $b(t)$

$$
b(t)=b_{m} \cdot \frac{c}{k_{\mathrm{off}} / k_{\mathrm{on}}+c} \cdot\left(1-e^{-\left(k_{\mathrm{on}} \cdot c+k_{\mathrm{off}}\right) \cdot t}\right)
$$

The impact of a reduction in $k_{\text {on }}$ is demonstrated by estimating how much additional time $t_{\text {add }}$ would be needed to reach saturation in a reaction $b_{\text {slow }}(t)$ with a lower $k_{\text {on }}$ than a reaction $b_{\text {ref }}(t)$ with a constant $k_{\text {on }}$. Assuming $k_{\text {on,ref }}=1 \cdot 10^{6} \mathrm{M}^{-1} \mathrm{~s}^{-1}$ for the reference reaction and $k_{\text {on,slow }}=0.5 \cdot 10^{6} \mathrm{M}^{-1} \mathrm{~s}^{-1}$ and an antibody concentration of $25 \mu \mathrm{L} / \mathrm{mL}$ for both reactions, we find that in the reaction with a lower $k_{\text {on }}$, surface saturation $(95 \%)$ is delayed by $\sim 110 \%$ in comparison to the reference case. 
As the variation in the performance of applied antibodies is found to have a large impact on the outcome of IHC assays, there is a strong need for a reliable way to validate antibodies under representative conditions to ensure consistency of results ${ }^{17}$.

\section{Materials and methods}

\section{Microfluidic probe head fabrication}

The microfluidic heads are hybrid Si/glass devices fabricated using a sequence of standard microfabrication steps: photolithography, deep reactive ion etching (DRIE), anodic bonding, and dicing. The design of the MFP heads was created in L-Edit V2016.2 (Tanner EDA), and the masks for photolithography were written on a laser writer (DWL 2000, Heidelberg Instruments, Germany). DRIE (AMS-200SE, Alcatel Micro Machining Systems, France) was performed on a double-side polished 4-inch $\mathrm{Si}$ wafer (500 $\mu \mathrm{m}$ thick, Siltronix, Geneva Switzerland) to pattern the microchannels, the through-etched apertures and the mesa of the MFP heads. The microchannels were etched to a depth of $50 \mu \mathrm{m}$. All three etching steps also contributed to patterning the outline of the hexagonal shape of the MFP heads. Si bridges were left to stand around the outline to keep the parts in place for further processing steps (more details in the SI). A 4-inch glass wafer $(1 \mathrm{~mm}$, Borofloat 33, SCHOTT AG, Germany) was used to fabricate the upper part of the head. Prior to anodically bonding the glass wafer to the patterned $\mathrm{Si}$ wafer, $500 \mu \mathrm{m}$ vias were drilled on a CNC milling machine (Wissner Gamma 303, Goettingen Germany) using a diamond drill (Haefeli AG, Switzerland). Wafer-level anodic bonding took place on a custom-made bonding machine $\left(475{ }^{\circ} \mathrm{C}\right.$, $1.3 \mathrm{kV})$. After dicing, the temporary $\mathrm{Si}$ bridges were removed using a scalpel blade.

\section{Fabrication and setting up the microfluidic probe station}

A custom holder was built to mount the MFP head and connect it to the pumping system (Fig. 2). The holder was assembled from several machined pieces and attached to the $z$-axis of an XYZ scanning system placed on an inverted microscope (Eclipse Ti, Nikon Instruments, Japan) (see SI1). The MFP head was mounted as follows: (1) The head was positioned in the holder (aluminum). (2) The PDMS gasket (molded from Sylgard ${ }^{\circledR} 184$ ) was placed on the head. The PDMS gasket self-aligned with the holder to match the holes to the via in the head. (3) A pressing block (PMMA) with holes for the capillaries, was placed on top of the gasket. The capillaries were inserted at the positions matching the microchannels in the head. (4) A bridge comprising a resilient pressure piece was mounted to the frame of the holder, and the resilient pressure piece was screwed towards the pressing block (finger-tight). This force compressed the gasket to lock the capillaries in place and ensure a leak-free connection (Figs. 2 and SI3).
With the MFP head mounted, the system was aligned to ensure coparallelism between the scanning stage $\mathrm{XY}$ movement, the substrate and the head surface by placing a dummy glass slide (Menzel, Germany) and adjusting the tilt of the head and substrate while establishing the zero Z-position at different positions on the slide repeatedly. Finding the zero Z-position was done by slowly moving the head towards the glass slide and observing the appearance of Newton's rings between the head's apex and the glass.

\section{Finite-element modeling}

We performed steady-state three-dimensional (3D) simulations with COMSOL Multiphysics (version 4.2). Nonslip boundary conditions were defined on all surfaces, constant flow velocity boundary conditions were applied at the aperture, and a constant pressure boundary condition was applied at the virtual interface between the liquid in the gap and the surrounding liquid at the edges of the apex. All fluids were designated as water (incompressible Newtonian fluid with a density of $998 \mathrm{~kg} / \mathrm{m}^{3}$ and a dynamic viscosity of $0.001 \mathrm{~N} \mathrm{~s} / \mathrm{m}^{2}$ ). The ratio of aspiration flow to injection flow was kept at 2.5, with an aspiration flow rate of $10 \mu \mathrm{L} / \mathrm{min}$.

\section{Micro-immunohistochemistry $(\mu \mathrm{HCC})$ protocol}

(1) Sample preparation: MDAMB468-1510A cell block sections were purchased from AMS Biotechnology Europe (Massagno, Switzerland). A standard protocol was applied to the cell block sections to prepare the sample for the IHC experiment. The preparation involved deparaffinization, hydrogen peroxidase block, target retrieval and protein block (see SI4 for more details on the protocol). The prepared cell block sections were kept in phosphate-buffered saline (PBS) at $4{ }^{\circ} \mathrm{C}$ for no longer than one day until use.

(2) MFP system setup: Two glass slides were placed into the substrate carrier next to each other. This allowed the fluidic system to be adjusted to achieve stable flow confinements on a dummy glass slide before performing experiments on the sample glass slide. The injection channels of the system were initially purged with colored liquids (diluted food colorant) until stable flow confinements were observed on the dummy glass slide. The pressure settings for each channel were saved in the control software to enable rapid switching between flow confinements with different reagents.

(3) $\mu \mathrm{IHC}$ : The MFP head was moved to the sample glass slide, which was covered with PBS, and positioned away from the cell block section at a safe distance (e.g., $15 \mathrm{~mm}$ ). The substrate-to-head 
gap distance was set to $75 \mu \mathrm{m}$. The reservoir tubes containing food colorant were replaced with tubes containing the reagents for IHC (primary antibody, secondary antibody and for some experiments streptavidin peroxidase and DAB substrate). The primary antibody (ab122898, anti-P53) as well as the other IHC chemicals (Mouse-specific HRP/ DAB (ABC) Detection IHC Kit, ab64259) were purchased from Abcam (Cambridge, UK). Each injection channel was purged with the corresponding IHC reagent until no residual food coloring could be observed. The pressure settings for stable flow confinements and switching between liquids were adjusted if needed. The MFP head was then moved to the $\mathrm{X}$ and $\mathrm{Y}$ zero positions in relation to the cell block section (upper left corner of an imaginary square fitted around the circular cell block section). This starting point for the experiment was set to 0 for the $x$ and $y$-axes in the control software.

(4) Design of experiments for $\mu \mathrm{IHC}$ conditions: Rapid $\mu \mathrm{IHC}$ with varying incubation times of the reagents was performed by moving to designated coordinates over the cell block section and switching between the reagent injection channels while keeping the aspiration flow constant. For scanning $\mu \mathrm{IHC}$, the MFP head was moved along the row of flow confinements at constant velocities. After applying the reagents with the MFP, the sample was processed for the subsequent steps according to a conventional IHC method. Depending on the number of reagents applied with the MFP, the cell block sections were incubated with streptavidin peroxidase and $\mathrm{DAB}$ substrate or with DAB substrate alone. No further treatment was needed for the cell block sections after scanning $\mu \mathrm{IHC}$. Processed cell block sections were dehydrated and mounted using standard mounting medium and a coverslip.

\section{Imaging and data processing}

An upright microscope (Eclipse 90i, Nikon Instruments, Japan) was used to image the sections, and color photographs were taken of each sample at $\times 4$ and $\times 10$ magnification. Quantification of the staining intensity was performed using ImageJ. Features were extracted manually, as this was considered sufficient for the conceptualization shown here. The individual images were analyzed as follows: (1) at least 25 nuclei per spot were selected with the freehand selection tool, and the mean gray value was calculated from all areas; (2) the same procedure was performed to calculate the mean gray value of the cytoplasm of the cells, where at least 25 regions per spot were selected manually for analysis. The normalized stain intensity per spot was then determined by subtracting the two values.

\section{Results and discussion \\ Horizontally oriented microfluidic probe heads for sequential chemistry}

In Lovchik et al. ${ }^{7}$, we demonstrated multiplexed $\mu \mathrm{IHC}$ on tissue sections within a single core of a tissue microarray. In that work, we implemented a single step of the $\mu \mathrm{IHC}$ process by locally presenting the primary $\mathrm{Ab}$ solution to the tissue with a vertically oriented MFP head using the basic configuration of an HFC formed via two square-shaped apertures. While this was a stride forward in performing local $\mu \mathrm{IHC}$, significant challenges remained: how to sequentially present multiple liquids to a small region of a tissue to implement a complete IHC protocol and how to increase the rapidity in the implementation of the complete protocol. The ability to switch between reagents can of course be extended to alternating between different primary antibodies for multiplexed analysis. In contrast, in this paper, we propose a new class of horizontally oriented MFP heads that not only allows the elegant implementation of efficient sequential protocols using multiple processing liquids but also provides flexibility in the placement of the apertures relative to each other and offers freedom in designing the apertures. The horizontal arrangement of the new generation of MFP heads provides room for the fluidic routing required for switching between different processing liquids and allows the deliberate shaping of apertures to enable the creation of different shapes of the confined liquids.

A challenge related to the horizontal orientation of the MFP heads is the resulting large surface area of the probe head. It increases the risk of damaging the sample due to improper alignment of the probe head with reference to the tissue section. We mitigate this issue by forming a $50 \mu \mathrm{m}$ high mesa at the center of the probe head, in which the apertures are placed and which is then closer to the sample, while the distance to the sample is larger for the remaining area of the probe head. There are several noteworthy aspects to the new head designs: $(i)$ Different flow confinement geometries are feasible on account of the flexibility of placement and shape of the apertures (square, circle, etc.). (ii) The circular arrangement of the apertures helps to minimize the cross-influence between adjacent HFCs, for which the earlier approach was to have additional stabilization apertures/flows, Taylor et al. ${ }^{12}$. (iii) Owing to central injection and side aspiration, the device can work in a more stable manner for larger substrate-to-head distances.

The spacing between the apertures was designed to minimize cross-talk between the different flow confinements when running simultaneously. This was done by performing finite-element simulations for both the circular and square-shaped HFCs (see Fig. 2c). An important 
aspect of the simulations was to ensure that the HFCs are in contact throughout the entire desired processing footprint on the sample. The simulations clearly showed that this is the case for gap distances between 50 and $100 \mu \mathrm{m}$, which is the range used in this work.

\section{Implementing sequential chemistry using the MFP}

We propose and implement two approaches to sequentially expose a surface to different reagents in order to implement a complete biochemical assay. One approach is to sequentially deliver liquids with the MFP positioned in a specific location over the surface. The second is to perform the sequential reagent exposure of the liquids to the surface by leveraging the scanning ability of the MFP in combination with several neighboring HFCs.

In the liquid switching sequential chemistry approach, the MFP head comprises three connectors to different liquids that merge into one injection aperture that opens into the center of the MFP head. By splitting the aspiration into four L-shaped structures, as seen on the bottom face of the head (see Fig. 3a), a square-shaped flow confinement is achieved. Here, we demonstrate sequential chemistry in a defined region by switching the flow of reagents externally. For this purpose, we control the flow of each individual injection channel. Since the merging of the three channels is close to the injection aperture, the dead volumes are extremely small $(<1 \mu \mathrm{L})$; therefore, the switching times for typical injection flow rates $(\sim 6 \mu \mathrm{L} /$ min) are just a few seconds. This number takes into account the exchange of the total liquid volume within the flow confinement $(0.02 \mu \mathrm{L})$. As the subsequent reagent completely displaces the previous liquid, intermediate washing steps, as applied in conventional protocols, are eliminated. This adds to the time advantage of this implementation. Using the approach of exchanging the liquids in the HFC, we performed anti-P53 staining on a $4 \mathrm{~mm}$ diameter cell block section (MDAMB468). We implemented three steps of the IHC protocol, wherein primary antibodies, secondary antibodies and streptavidin peroxidase are displayed to the cell block section sequentially. As seen in Fig. 3b, we stained footprints of $\sim 500 \times 500 \mu^{2}$, and in each of these footprints, we varied the incubation times of the three steps (incubation times provided in Fig. SI.5). As expected, the signal intensities differed depending on the incubation strategies, and good contrast and uniformity were obtained even with incubation times as low as $30 \mathrm{~s}$ for the primary antibody.

Another approach to the rapid sequential exposure of multiple liquids on a surface is to scan with an MFP multiple HFCs along one axis over the surface. We designed a specific MFP head, where five HFCs can be run simultaneously in a linear arrangement, each confining a different liquid. Figure 3c, d show the experimental performance using four different chemicals, with the fifth port left unused. The size of the HFCs and the scanning velocity define the incubation times for the reagents on the surface of the sample. Note that the incubation time of an individual reagent could also be adapted through the chip design or by confining the same reagent in multiple neighboring HFCs. The injection channels in this configuration do not merge in a single injection aperture as in the other chip design. This is an advantage because two sequential reagents could potentially react and clog the channel. As each flow confinement is $500 \mu \mathrm{m}$ wide, and these confinements are linearly arranged during scanning, the resulting stained tissue section appears as a line along the $y$-axis, Fig. $3 \mathrm{~d}$. In addition, the separation of the HFCs prevents cross-contamination of reagents because there is a small amount of immersion liquid between all HFCs, as illustrated in the bottom of Fig. 2c. The scanning approach also led to good staining results with an incubation time of $30 \mathrm{~s}$ for each of the four implemented steps.

\section{Towards optimizing processing parameters for establishing rapid and efficient IHC}

In conventional $\mathrm{IHC}$, the practice is to make use of protocols that previously provided good results for a given type of tissue, antigen of interest and primary antibody. Predefined protocols, however, do not take into account the expression level of the antigens in a specific sample or batch-to-batch variations in the performance of the applied antibodies. A simple and rapid way to first test the IHC parameters for a given sample would, therefore, allow optimization of the quantity and the quality of the information that can be retrieved from an IHC analysis. It is possible to perform such optimization using entire tissue sections. However, to traverse a reasonable number of process parameters, the amount of tissue required to determine an ideal set of processing parameters will be rather large. As the MFP allows IHC to be performed at the micrometer length scale, a fraction of the section can be dedicated to such optimization purposes. Alternatively, instead of using fractions of several sections, a single section can be dedicated to establishing suitable IHC process parameters. While this idea is well suited for diagnostics, it is even more suitable for optimizing conditions for screening-like applications, such as those performed with tissue microarrays.

To demonstrate this concept of process parameter optimization, we made use of another cell block section to demonstrate sequential IHC. To determine the ideal incubation times for the primary and secondary antibody solutions, we designed experiments (central composite design) in which these two factors were systematically varied (see Fig. SI.6). The experimental design consisted of independent sequential reactions performed on 12 spots of the same cell block section. To ensure that there was no nonspecific binding of the antibodies, we 


\section{a}

Liquid switching sequential chemistry

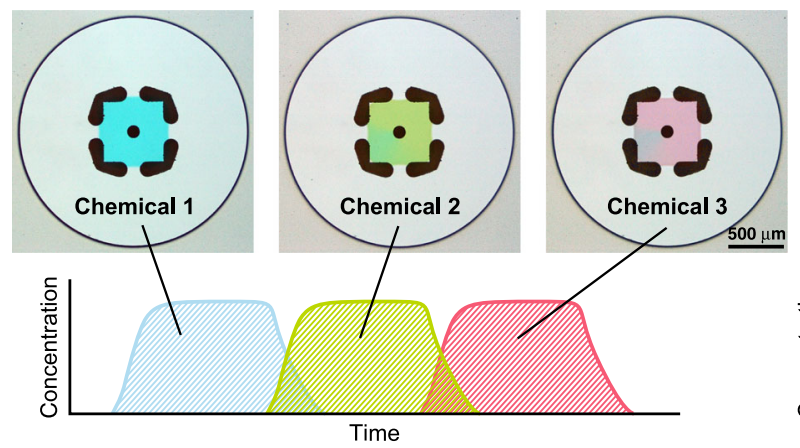

b

Liquid switching $\mu \mathrm{HHC}$

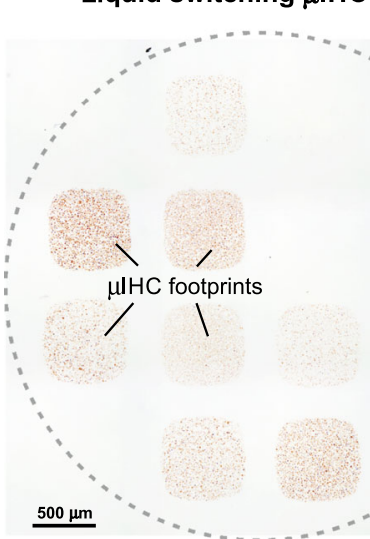

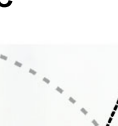
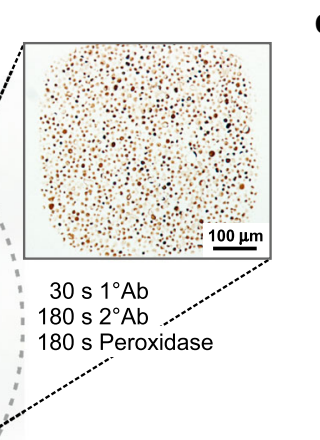

C

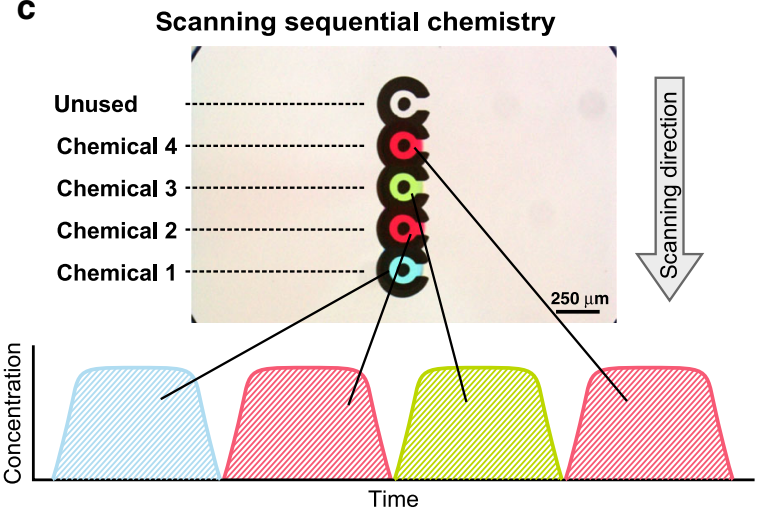

d

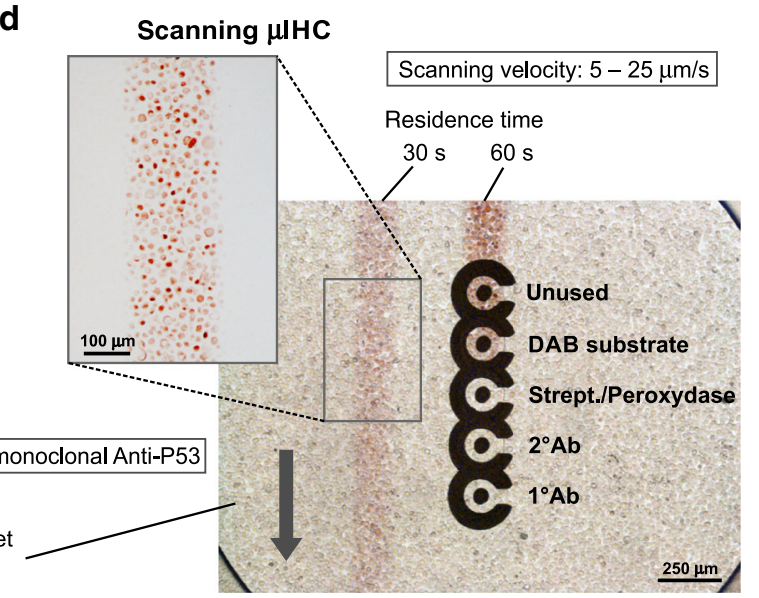

Fig. 3 Experimental results of two implementations of sequential chemistry to perform the complete IHC protocol on cell blocks. $\mathbf{a}$ and $\mathbf{b}$ involve the static head, while $\mathbf{c}$ and $\mathbf{d}$ involve the scanning head. a Photographs of a single HFC captured at three different times with different colored liquids. The liquids within the HFC can be switched within $\sim 2 \mathrm{~s}$. b $\mathrm{HC}$ staining for P53 on a cell pellet section with varying incubation times of the primary Ab, secondary Ab, and streptavidin peroxidase at different locations. c Scanning of the reagents with a multi-HFC head allows fast sequential chemical treatment of a surface. This strategy was also applied for $\mathrm{HC}$ on a cell pellet section (d), and good contrast was achieved with residence times as short as $30 \mathrm{~s}$

implemented negative controls without primary or secondary antibodies on two additional spots (bottom left and right spot in Fig. 4a). A selection of higher-resolution images can be found in the SI, Fig. SI.7.

After spotwise sequential incubation, streptavidin peroxidase and DAB chromogen were applied over the entire section. Even with highly variable incubation times, stained areas may appear very similar at first glance. However, it is essential to determine the difference in staining intensities between the nuclei and the cytoplasm to extract meaningful quantitative data. An analysis of the results revealed significant first- and second-order effects of the incubation time with primary and secondary antibodies (Fig. 4b). While increasing either of the two incubation times had a positive effect on the signal level, the signal contrast deteriorated for longer incubation with primary antibodies. Our results, therefore, suggest that for the current set of antibodies/antigens, an optimized workflow should have a short incubation time for the primary antibody and an incubation time approximately five times longer for the secondary antibody. While the general idea of having minimal incorrect binding in the first step (primary antibody) and high amplification in the second step holds, the exact ratio of incubation times during each step might vary and would need to be established on a case-by-case basis.

\section{Summary and concluding remarks}

The presented strategy for rapid IHC analysis is conservative in the consumption of a tissue section, benefits from reduced amounts of reagents and is able to screen the process parameters of the IHC protocol for good quality stains. This approach complements our previous work on computational heuristics to establish process parameters for high-quality staining ${ }^{18,19}$. The core of performing such microscale IHC reactions on a tissue section is the hydrodynamic confinement of liquids using the microfluidic probe. Here, we specifically propose two designs of MFP 


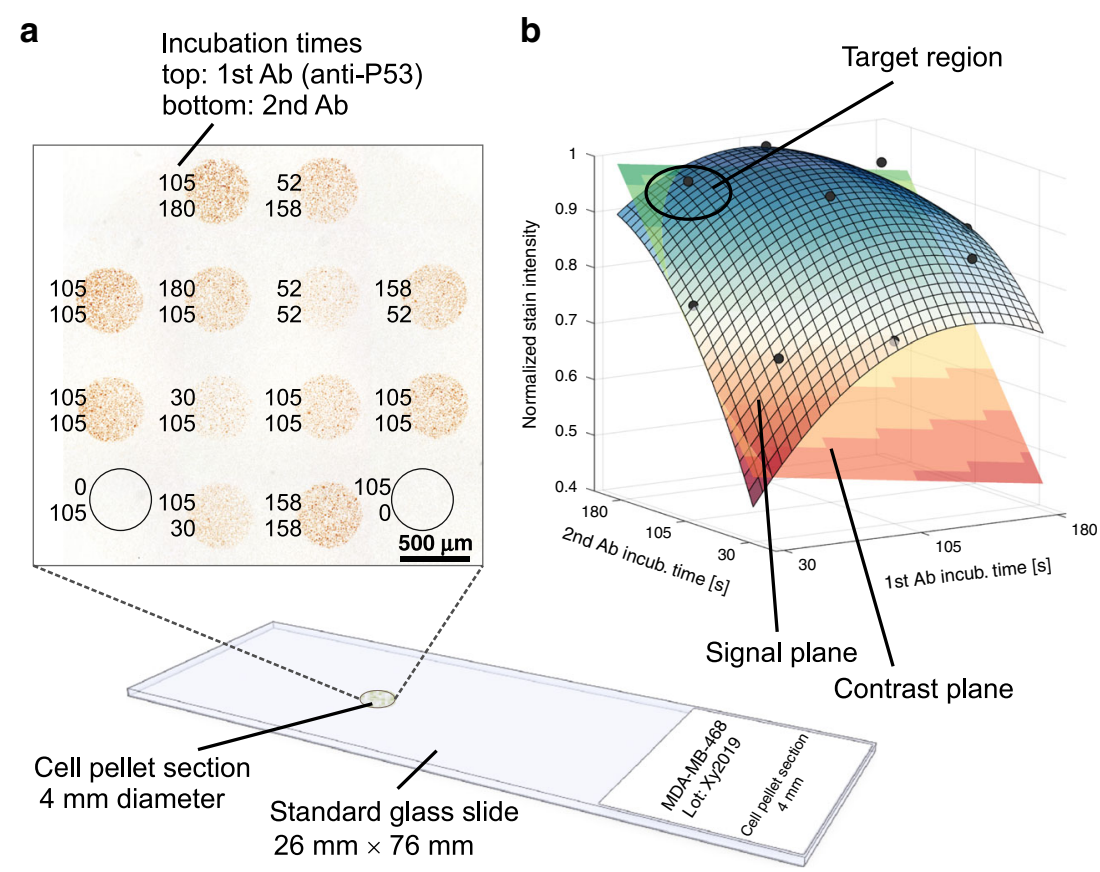

Fig. 4 Exploring suitable staining conditions using process parameter optimization. a Experimental result of a two-factor central composite design with two negative controls on a single cell block section (incubation times shown in seconds). Streptavidin peroxidase and DAB chromogen were applied globally. $\mathbf{b}$ Analysis reveals statistically relevant first- and second-order effects for both factors. The figure displays the response surfaces for the signal intensity (red-white-blue) and for the signal contrast (red-yellow-blue). The negative curvature of the intensity response surface for long incubation times with primary antibodies does not make sense physically and is considered an artifact, which could be removed by increasing the number of datapoints. The signal contrast deteriorates for long incubation with the primary antibody. Therefore, relatively short incubation with primary antibodies and relatively long incubation with secondary antibodies results in the highest signal at the best contrast

heads to implement a complete IHC protocol. The implementation of the $\mu \mathrm{IHC}$ protocol is rapid in the first case through the continuous replenishment of the reagents on the tissue surfaces due to the flows inherent to the $\mathrm{MFP}^{20}$ and in the second case due to the sequential presentation of reagents, which also simultaneously rinse out the previous liquid. From a technical perspective, this new class of MFP heads provides new opportunities for how liquids can be presented to the surface and how explicit shapes of the confined liquid can be formed.

Owing to the above attributes, we think this may be the genesis of real-time processing of tissue sections with an immediate readout using nonfluorescent labels, resulting in compatibility with existing pathology workflows. The devices and methods presented build on established and robust workflows while significantly enhancing their dynamics and versatility. Additionally, real-time IHC staining can be readily applied to create gradients of the primary antibody, thus enabling quantitative $\mathrm{IHC}^{21}$. The strategies presented here can be used for whole tissue processing. Furthermore, to capture the variations in the tissue and support statisticsguided decision-making, we think image processing software would facilitate the implementation of MFP-based IHC in fast and automated analysis routines. The presented methods may also be extended to include preanalytical steps required prior to staining (such as dewaxing and antigen retrieval treatments).

Another interesting direction of this approach is to study the kinetics of an antibody-antigen reaction. It is well known that applying mathematical models does not allow accurate establishment of the association constants of the applied antibodies. Nonetheless, our method for the local implementation of a specific combination of staining conditions reveals the impact of varying $k_{\text {on }}$ and compares the performance of different batches to optimize protocols in a targeted manner. The incubation times of primary and secondary antibodies can be varied systematically following a design of experiments and creating arrays of stains representing different incubation conditions. In this work, we implemented a full factorial design to assess the effects of the incubation times with the primary antibody and the secondary antibody. An array of stains featuring permutations of different incubation times with the primary and secondary antibody solutions would allow us to compare antibodies from different batches or vendors and to optimize incubation times in staining protocols in a targeted manner. In summary, our technology can be used for quality checks 
and screening, and we think that it has the potential to deepen insights into valuable tissues and increase the information obtained in scientific studies. Moreover, we see great potential in implementing rapid IHC in scenarios where time to result is highly critical.

The current development stage of the MFP heads and the experimental platform still has some limitations. The stability of the liquid flow and switching between liquids are sensitive to air bubbles forming in the tubing or channels. Although degassing of liquids and good practice in setting up the experiments minimizes the occurrence of bubbles and consequent failure of the experiment, there is still a need for further engineering of the system. There are several strategies in microfluidics to avoid or cope with bubble formation ${ }^{22}$. We are also aware that the current design is complex for fabrication, especially the $\mathrm{Si}$ /glass hybrid heads, which require microfabrication in a clean room. We are currently translating several of the MFP head designs to work in plastics and become suitable for injection molding.

In summary, we think that there is a need for strategies in pathology to enable preoperative molecular screening of tissues while using as much of the current workflows as possible. IHC, due to its complex multistep and sometimes even multiday protocols, is confined to central laboratories. Increasing its rapidity and automation could enable the use of this method for diagnostics on fresh tissue in combination with surgery. We believe that, although still rudimentary, the methodology presented here provides a starting point towards closing that gap while enabling high-quality quantitative IHC to reduce errors in diagnostics. To optimize our method for use in a clinical setting, further validation by pathologists is required. Such input would help to determine, for example, the value of the time advantage for multiplexed tissue analyses and whether the size of the stained regions is sufficient for diagnostic interpretation. Overall, the attributes of the methodology could also help significantly in addressing open research questions, such as probing tumor heterogeneity.

\section{Acknowledgements}

We thank Anna Fomitcheva Khartchenko, Aditya Kashyap, and Lena Voith von Voithenberg for providing support during the experiments and Linda Rudin for critical comments on the manuscript. We thank Ute Drechsler and Diana Davila Pineda for help with the fabrication of the probe heads. We acknowledge Emmanuel Delamarche and Heike Riel for their constant support. This work was supported by the European Research Council PoC Grant CellProbe (842790).

\section{Author contributions}

R.D.L. designed and fabricated the MFP heads and performed the wet-bench experiments. D.T. performed the design of experiments analysis. R.L., D.T., and G.V.K designed the overall work and wrote the manuscript.
Conflict of interest

The authors declare that they have no conflict of interest.

Supplementary information accompanies this paper at https://doi.org/ 10.1038/s41378-020-00205-2.

Received: 31 January 2020 Revised: 6 August 2020 Accepted: 7 August 2020

Published online: 19 October 2020

\section{References}

1. Bradbury, A. \& Plückthun, A. Reproducibility: standardize antibodies used in research. Nature 518, 27-29 (2015).

2. Dupouy, D. G. et al. Continuous quantification of HER2 expression by microfluidic precision immunofluorescence estimates HER2 gene amplification in breast cancer. Sci. Rep. 6, 20277 (2016).

3. Carvajal-Hausdorf, D. E., Schalper, K. A., Neumeister, V. M. \& Rimm, D. L. Quantitative measurement of cancer tissue biomarkers in the lab and in the clinic. Lab. Investig. 95, 385-396 (2015).

4. Xing, Y. et al. Bioconjugated quantum dots for multiplexed and quantitative immunohistochemistry. Nat. Protoc. 2, 1152-1165 (2007).

5. Kashyap, A. et al. Quantitative microimmunohistochemistry for the grading of immunostains on tumour tissues. Nat. Biomed. Eng. 3, 478-490 (2019).

6. Gannot, G. et al. Layered peptide arrays: High-throughput antibody screening of clinical samples. J. Mol. Diagnostics 7, 427-436 (2005).

7. Lovchik, R. D., Kaigala, G. V., Georgiadis, M. \& Delamarche, E. Microimmunohistochemistry using a microfluidic probe. Lab Chip 12, 1040 (2012).

8. Kim, M. S. et al. Breast cancer diagnosis using a microfluidic multiplexed immunohistochemistry platform. PLOS ONE 5, e10441 (2010).

9. Ciftlik, A. T., Lehr, H.-A. \& Gijs, M. A. M. Microfluidic processor allows rapid HER2 immunohistochemistry of breast carcinomas and significantly reduces ambiquous (2+) read-outs. Proc. Natl Acad. Sci. USA 110, 5363-5368 (2013).

10. Kaigala, G. V., Lovchik, R. D., Drechsler, U. \& Delamarche, E. A vertical microfluidic probe. Langmuir 27, 5686-5693 (2011).

11. Queval, A. et al. Chamber and microfluidic probe for microperfusion of organotypic brain slices. Lab Chip 10, 326-334 (2010).

12. Taylor, D. P., Zeaf, I., Lovchik, R. D. \& Kaigala, G. V. Centimeter-scale surface interactions using hydrodynamic flow confinements. Langmuir 32, 10537-10544 (2016)

13. Song, B. et al. Time-resolved lanthanide luminescence for lab-on-a-chip detection of biomarkers on cancerous tissues. Analyst 134, 1991-1993 (2009).

14. Cappi, G., Dupouy, D. G., Comino, M. A. \& Ciftlik, A. T. Ultra-fast and automated immunohistofluorescent multistaining using a microfluidic tissue processor. Sci. Rep. 9, 4489 (2019).

15. Cors, J. F., Kashyap, A., Khartchenko, A. F., Schraml, P. \& Kaigala, G. V. Tissue lithography: microscale dewaxing to enable retrospective studies on formalinfixed paraffin-embedded (FFPE) tissue sections. PLOS ONE 12, e0176691 (2017).

16. Bordeaux, J. et al. Antibody validation. BioTechniques 48, 197-209 (2010).

17. Baker, M. Blame it on the antibodies. Nature 521, 274-276 (2015).

18. Arar, N. M. et al. Computational immunohistochemistry: recipes for standardization of immunostaining, In Lecture Notes in Computer Science. Vol. 10434 (International Conference on Medical Image Computing and ComputerAssisted Intervention (MICCAI), Quebec City, QC, Canada, 2017). https://doi. org/10.1007/978-3-319-66185-8_6.

19. Arar, N. M. et al. High-quality immunohistochemical stains through computational assay parameter optimization. IEEE Trans. Biomed. Eng. 66, 2952-2963 (2019).

20. Autebert, J., Cors, J. F., Taylor, D. P. \& Kaigala, G. V. Convection-enhanced biopatterning with recirculation of hydrodynamically confined nanoliter volumes of reagents. Anal. Chem. 88, 3235-3242 (2016).

21. Kashyap, A., Autebert, J., Delamarche, E. \& Kaigala, G. V. Selective local lysis and sampling of live cells for nucleic acid analysis using a microfluidic probe. Sci. Rep. 6, srep29579 (2016)

22. Pereiro, l., Fomitcheva Khartchenko, A., Petrini, L. \& Kaigala, G. V. Nip the bubble in the bud: a guide to avoid gas nucleation in microfluidics. Lab Chip 19 2296-2314 (2019). 